\title{
Application of genetic algorithm to the optimization of resonant frequency of coaxially fed rectangular microstrip antenna
}

\author{
${ }^{1}$ Ruchi Varma, ${ }^{1}$ Serene Bhaskaran, ${ }^{2}$ Jayanta Ghosh \\ ${ }^{I}$ (ECE, National Institute of technology, Patna, India) \\ ${ }^{2}$ (Assistant professor, ECE, National Institute of technology, Patna, India)
}

\begin{abstract}
Microstrip antenna is gathering a lot of interest in communication systems. Genetic algorithm is a popular optimization technique and has been introduced for design optimization of microstrip patch antenna. In this paper, genetic algorithm has been used for optimization of resonant frequency of coaxially fed rectangular microstrip antenna. The investigation is made at 3 different frequencies $3 \mathrm{GHz}, 5 \mathrm{GHz}$ and $10 \mathrm{GHz}$ respectively. Patch length, patch width \& feed position are taken as optimization parameters. Return loss and radiation pattern for the optimized antenna are verified using IE3D software. Accuracy of the results encourages the use of genetic algorithm.
\end{abstract}

Keywords - Rectangular microstrip antenna, genetic algorithm, resonant frequency, IE3D.

\section{Introduction}

Wireless communication systems are in use in multitude of sizes ranging from small hand held devices to devices mounted on vehicles. For optimum system performance, high radiation efficiency, small volumes, simple \& low loss impedance matching to receive and transmit paths are necessary prerequisites of the antennas. Micro strip antennas are appropriate candidates to meet the mentioned requirements Micro strip antennas are expected to find many promising applications in wireless communications because of their attractive merits of low profile, light weight, ease of fabrication, and good conformability with integrated circuits. [1]

One of the drawbacks of the microstrip antennas is their narrow bandwidth. As a consequence, antennas can work efficiently only close to their resonance frequency. For this reason, the accurate evaluation of this parameter is of fundamental importance.

In recent years several algorithms have been developed for optimization of various kinds of problem related to antenna design. The aim of any optimization technique is to find a solution that represents a global maximum or minimum in a suitably defined solution domain, that means to find the best solution among many possible solutions for a considered problem. [2]

This paper presents a method for resonant frequency optimization of coaxially fed rectangular microstrip antenna using genetic algorithm. The optimization problem has three variables namely patch length, width and feed position respectively.

\section{Theory}

A microstrip patch antenna consists of a very thin metallic patch (usually gold or copper) placed a small fraction of a wavelength above a conducting ground plane, separated by a dielectric substrate.[3] There are various feeding techniques used for feeding microstrip patch antennas such as microstrip line, coaxial probe, aperture coupling and proximity coupling. In this paper, patch is fed by a coaxial feed as shown in Fig.1.

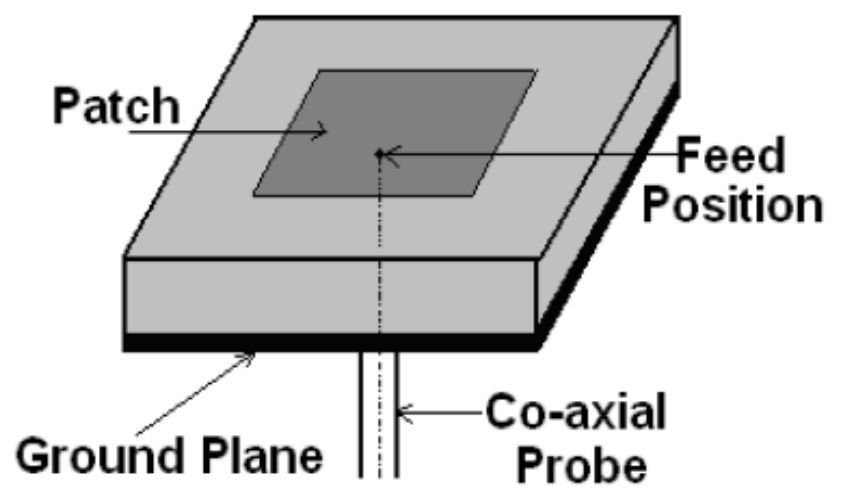

Fig.1. Probe-fed microstrip antenna 
The transmission line model and cavity model are approximate models often used for design and analysis of microstrip antennas. Here we have used the cavity model for our analysis.

The rectangular microstrip patch antenna can be considered in the fundamental mode, modelled by a simple resonant parallel RLC circuit, as shown in Fig.2. In order to take the coaxial-feed probe into account, it is necessary to modify the input impedance by an inductive reactance term $\left(\mathrm{X}_{\mathrm{L}}\right)$.

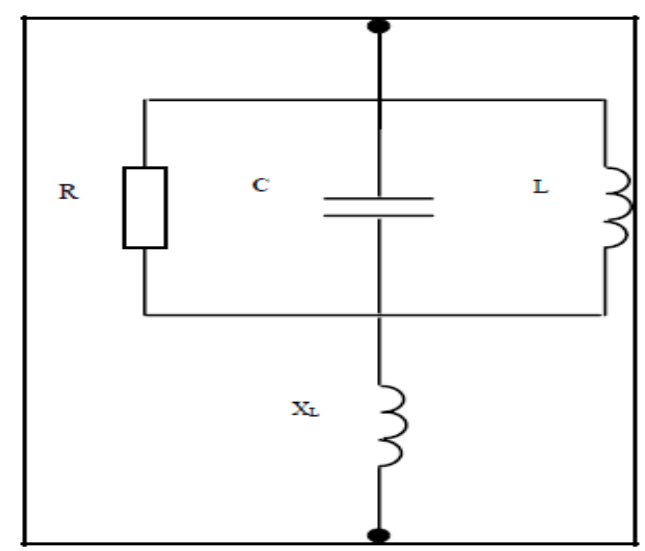

Fig.2. Equivalent resonant parallel RLC circuit

Thus the input impedance of coaxially fed microstrip antenna is given as:

$$
\operatorname{Zin}=\frac{R}{1+Q 2\left(\frac{f}{f_{r}}-\frac{f r}{f}\right)^{2}}+j\left(X l-\frac{\frac{R}{1+Q\left(\frac{f}{f r}-\frac{f r}{f}\right)}}{\frac{R}{1+Q} 2\left(\frac{f}{f_{r}}-\frac{f r}{f}\right)^{2}}\right)
$$

where, $\mathrm{R}$ is the resonant resistance of the resonant parallel RLC circuit, fr is the resonant frequency, and $\mathrm{Q}$ is the total quality factor associated with system losses including radiation, the loss due to heating in the conducting elements and the ground plane, and the loss due to heating within the dielectric medium.

\section{Genetic Algorithm}

A genetic algorithm has been developed for determining the parameters to provide accurate value of the resonant frequency. Genetic algorithm is a robust global stochastic search methods based on the Darwinian concepts of natural selection and evolution. The parameters of each individual of the population are usually encoded as a string of bits (chromosomes). The first group of individuals (generation) is created randomly. The fitness of each individual is determined. Mating these individuals forms a new generation. The more fit individuals are selected and given greater chance of reproducing. Crossover and mutation are used to allow global exploration of the cost function. The best individual may be passed unchanged to the next generation. This iterative process creates successive generations until a stop criterion is reached. It is expected that individuals of successive generations converge to the global maximum. [4], [5].

\subsection{Genetic Algorithm Based Optimization Of Resonant Frequency}

In this paper, coaxially fed microstrip antenna is considered for optimization. The fitness function is defined as: $\mathrm{F}=(\operatorname{abs}(\mathrm{f}-\mathrm{fr})+\operatorname{abs}(\operatorname{real}(\mathrm{Z})-50)+\operatorname{abs}(\operatorname{imag}(\mathrm{Z})-0))$

where $\mathrm{Z}$ is an input impedance and $\operatorname{real}(\mathrm{Z})$ is the real part \& imag(Z)is the imaginary part of $\mathrm{Z}$.

At resonant frequency imaginary part of input impedance should be zero. Impedance matching is a very important factor and must be considered for designing an antenna. Perfect impedance matching leads to maximum coupling and thus minimizing the return loss and return loss at resonant frequency can be minimized if real part of input impedance is close to $50 \Omega$.

The genetic algorithm is used for optimizing operations. It performs three tasks as follows:

1) It generates the parameters of each structure and sends them to the fitness module.

2) It receives each structure's fitness value from the fitness module. 
3) Based on the fitness values, the next generation is created by a reproduction process that involves roulette wheel selection, crossover, mutation, and elitist strategy.

The reproduction process is iterated until the fitness function converges to a maximum value, or the termination criterion is met. [6]

In this paper probability of crossover \& probability of mutation are taken as $0.8 \& 0.05$ respectively. The process is repeated for 50 iterations which is the criterion for termination of the process.

\section{Results And Discussions}

The investigation is made at three different frequencies namely $3 \mathrm{GHz}, 5 \mathrm{GHz}$ and $10 \mathrm{GHz}$ respectively. Table 1 tabulate the optimized values of patch length patch width and feed position for different frequencies. Figs.3-6 show the simulated return loss and radiation pattern plots at different resonant frequencies, for each case.

Table 1

\begin{tabular}{|c|l|l|l|l|}
\hline $\begin{array}{l}\text { Resonant frequency } \\
(\mathrm{GHz})\end{array}$ & $\begin{array}{l}\text { Optimum patch } \\
\text { length } \\
(\mathrm{mm})\end{array}$ & $\begin{array}{l}\text { Optimum patch } \\
\text { width } \\
(\mathrm{mm})\end{array}$ & $\begin{array}{l}\text { Feed position } \\
(\mathrm{mm})\end{array}$ & $\begin{array}{l}\text { Return loss } \\
(\mathrm{db})\end{array}$ \\
\hline 3 & 22.9 & 43.5 & 8 & -18.7091 \\
\hline 5 & 18.325 & 31 & 6 & -22.0256 \\
\hline 10 & 6.25 & 14.8 & 2 & -41.752 \\
\hline
\end{tabular}

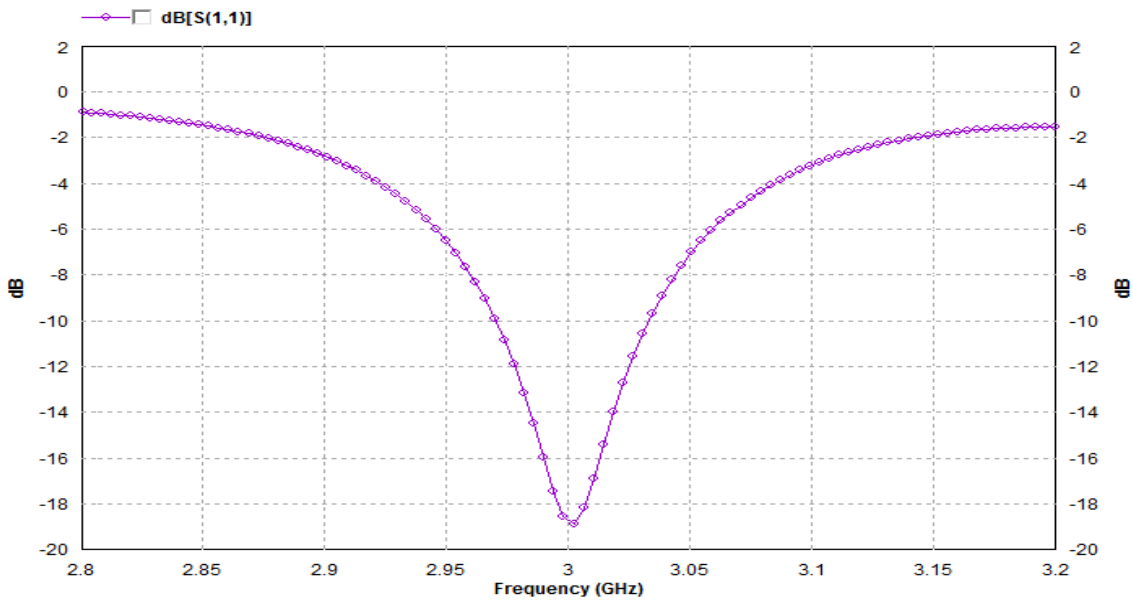

Fig.3. Return loss plot for frequency $3 \mathrm{GHz}$

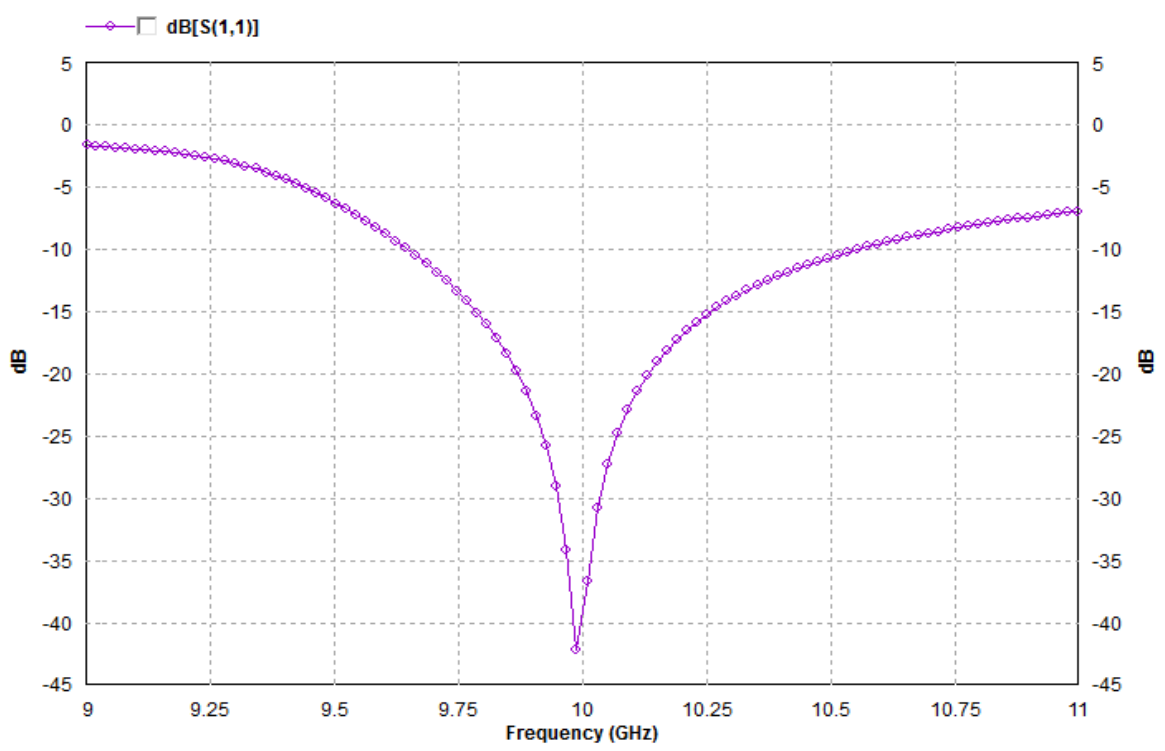

Fig.4. Return loss plot for $10 \mathrm{GHz}$ resonant frequency 


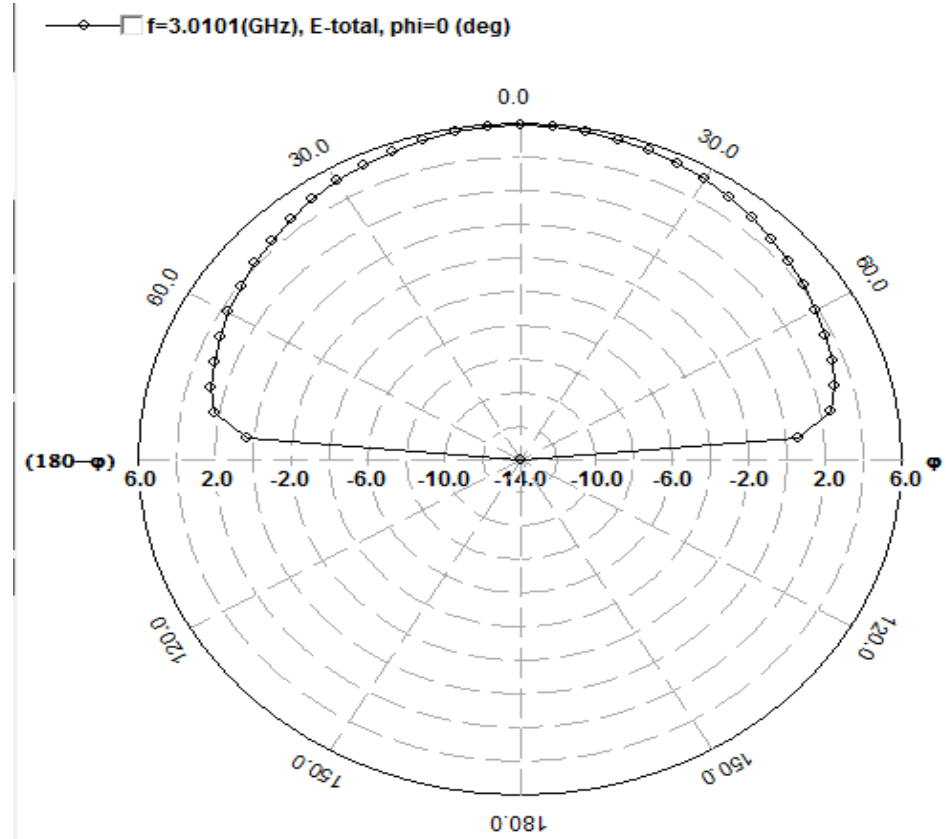

Fig.5. Radiation pattern for frequency $3 \mathrm{GHz}$

$\longrightarrow-\Gamma \mathrm{f}=10.0101(\mathrm{GHz}), \mathrm{E}$-total, phi $=0(\mathrm{deg})$

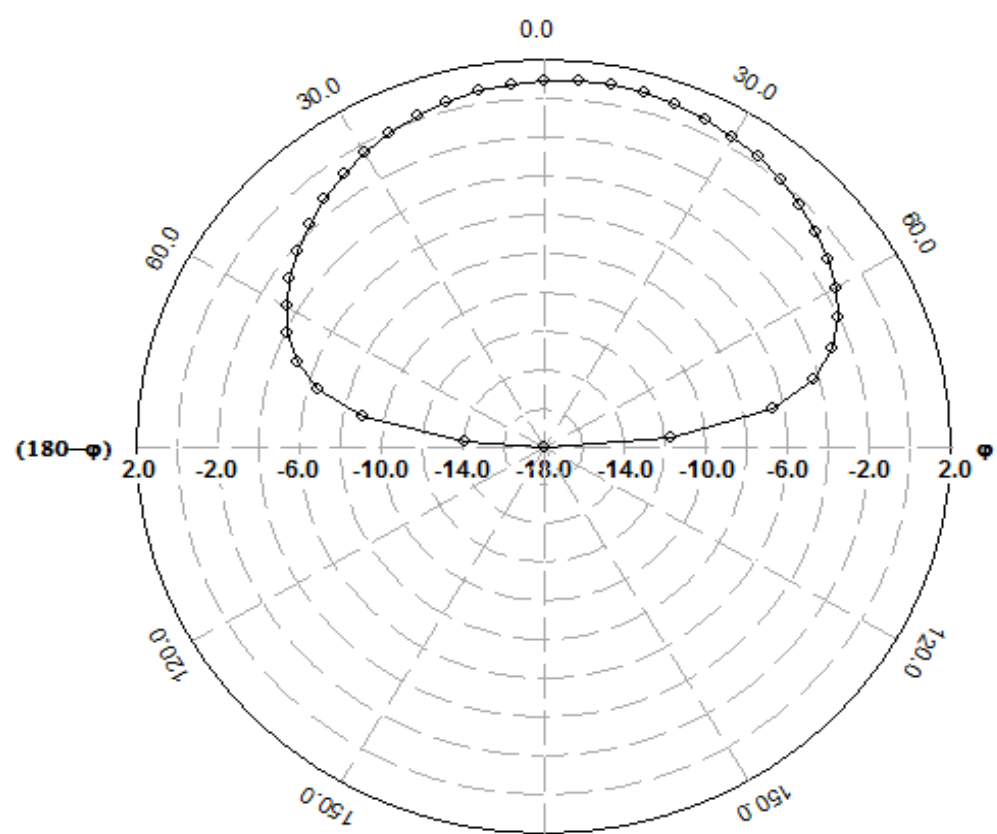

Fig.6. Radiation pattern for frequency $10 \mathrm{GHz}$

\section{Conclusion}

The method of designing microstrip antenna, using genetic algorithm, is presented in this paper. The accurate evaluation of resonance frequency of microstrip antennas is a key factor to guarantee their correct behaviour. Thus genetic algorithm has been used for optimization of resonant frequency for perfect impedance matching. Results are verified by IE3D software. The return loss plots show that the resonant frequencies obtained from the optimization process exactly matches with the desired values. The radiation pattern clearly shows that there is radiation only in the forward direction but no radiation in the backward direction which gives an accuracy of the impedance matching of the designed antenna. This means that the antenna is perfectly matched. Results encourage the use of genetic algorithm for optimal design. 


\section{Acknowledgements}

This work is supported by Electronics and Communication Department, National Institute of Technology, Patna, India as a part of partial fulfilment of post graduate degree in communication systems.

\section{References}

[1] R. Garg, P. Bhartia, I. J. Bahl, and A. Ittipiboon, Microstrip Antenna Design Handbook. Artech House, (2001).

[2] A. Torn and A. Zilinskas, Global Optimization. New York: Springer-Verlag, (1989), vol. 350, Lecture Notes in Computer Science.

[3] Constantine A. Balanis,' Antenna Theory: Analysis Design', Third Edition, by ISBN 0-471-66782-X Copyright (2005) John Wiley \& Sons, Inc

[4] R. L. Haupt and S. E. Haupt, Practical Genetic Algorithms. New York: Wiley( 1998).

[5] Y. Rahmat-Samii and E. Michielssen, Electromagnetic Optimization by Genetic Algorithms, Y. Rahmat-Samii and E. Michielssen, Eds. New York: Wiley, (1999).

[6] Siyang SUN, Yinghua LU, Jinling ZHANG, Fangming RUAN, Genetic algorithm optimization of broadband microstrip antenna. 